\title{
¿Es curable el cáncer de mama en etapa precoz? Resultados del tratamiento combinado con cirugía, radioterapia y quimioterapia
}

\author{
César Sánchez $R^{1}$, Marisa Bustos $C^{3}$, Mauricio Camus $A^{2}$, \\ Manuel Álvarez $Z^{1}$, Ignacio $G$ oñi $E^{2}$, Augusto León $\mathbf{R}^{2}$, \\ Pelayo Besa de $\mathrm{C}^{3}$.

\section{Treatment results of early breast cancer. A retrospective review}

Background: Breast cancer will develop in one out of ten women during their lifetime. Early diagnosis has increased in recent years. Aim: To describe a population of women with breast cancer stage T1N0M0. To analyze radiation therapy toxicity and to evaluate treatment results. Material and methods: Retrospective review of the medical records of 125 women (aged 35 to 80 years) with breast cancer T1N0M0, that were treated between January 1997 and May 2004, with breast conserving surgery and postoperative radiation therapy at an oncology center. Patients lost from follow up were contacted by telephone. Results: An abnormal screening mammography was the reason for consult in $62 \%$ of cases. The average tumor size was $11.6 \mathrm{~mm}$. Tumors detected with screening mammogram were smaller than those detected on physical exam. The most common radiotherapy toxicity was erithema, which was severe in $2.5 \%$ of cases. No patient had to stop the radiation treatment due to toxicity. One patient developed arm edema. Tamoxifen was prescribed for 5 years to $80 \%$ of patients and 17 patients received chemotherapy. After an average follow up of 40 months, no patient has developed local breast relapse, three patients developed contralateral breast cancer and three developed distant metastasis. Two patients died from breast cancer. Disease free survival was 95\%. Conclusions: Radiotherapy was well tolerated and had excellent local control. Screening mammography detects small tumors. Survival is excellent for early stage breast cancer (Rev Méd Chile 2007; 135: 427-35).

(Key-w ords: Breast neoplasms; Mammography; Radiotherapy; Tamoxifen)

Recibido el 23 de enero, 2006. Aceptado el 22 de septiembre, 2006.

${ }^{1}$ Departamento de Hematología-Oncología. ${ }^{2}$ Departamento de Cirugía. ${ }^{3}$ Departamento de Radiología. Programa de Cáncer, Facultad de Medicina, Pontificia Universidad Católica de Chile.

Correspondencia a: Dr. Pelayo Besa de C. Centro de Cáncer, Pontificia Universidad Católica de Chile. Diagonal Paraguay 319, Santiago, Chile. Fono: 56-2-3546919. Fax: 562-24723327. E mail: pbesa@med.puc.cl 
A lo largo de su vida, una mujer tiene la posibilidad de 1 en 10 de desarrollar cáncer de mama ${ }^{1}$. Las neoplasias de mama ocuparán la primera frecuencia en la incidencia de nuevos casos de cáncer en Estados Unidos de Norteamérica el año 2006 con 31\% del total, sobre el cáncer de pulmón y bronquios, y será la segunda causa de muerte por cáncer en mujeres ${ }^{2}$. En Chile, el cáncer de mama ocupa la segunda causa de muerte por neoplasias en el sexo femenino, luego del cáncer de vesícula y vía biliar3,4.

En las últimas décadas, la frecuencia de tumores pequeños al momento del diagnóstico ha aumentado. Entre $30 \%$ y $50 \%$ de los tumores son diagnosticados mamográficamente y hasta 30\% pueden medir menos de $1 \mathrm{~cm}^{5}$; en la década de 1960-69, $80 \%$ de los tumores eran detectados al examen físico y medían más de $2 \mathrm{~cm}^{6,7}$. En Chile, en el año 2003, del total de casos de cáncer de mama evaluados en el sistema público de salud, 14\% correspondió a tumores en etapa $\mathrm{I}^{3}$. El cambio epidemiológico en la presentación del cáncer de mama, cada vez con mayor incidencia de tumores pequeños, sin compromiso linfático, implica un gran desafío para definir la oportunidad y la indicación del tratamiento local y sistémico.

La evaluación inicial de la paciente incluye la etapificación de la enfermedad. El sistema universalmente aceptado es la clasificación TNM; ésta utiliza variables anatómicas del tumor, relacionadas al pronóstico de la enfermedad, propuesta por el American Joint Committee on Cancer (AJCC) ${ }^{8,9}$ (Tabla 1).
La medición de estas variables identifica a pacientes de acuerdo al riesgo de mortalidad y recurrencia de la enfermedad.

De este modo, tumores pequeños de hasta 2 $\mathrm{cm}$ de diámetro mayor (T1), sin compromiso linfático (N0) y en ausencia de enfermedad metastásica (M0), tienen un mejor pronóstico en comparación con tumores en estadios más avanzados. A pesar de la ausencia de compromiso linfático, estos tumores pueden tener recurrencias locales hasta en $20 \%-30 \%$, cuando son tratados sólo con cirugía $^{10,11}$. La radioterapia ha mostrado disminuir las recurrencias locales del cáncer de mama luego de una mastectomía parcial en cerca de $70 \%{ }^{11}$. El protocolo NSABP B-06 comparó resultados en un grupo de pacientes tratados con sólo mastectomía parcial versus aquellos tratados con mastectomía parcial más radioterapia versus mastectomía radical modificada. A 20 años, la recurrencia fue de $39 \%$ en el grupo tratado con sólo mastectomía parcial y de $14 \%$ en los pacientes que recibieron mastectomía parcial más radioterapia adyuvante ${ }^{12}$.

Se entiende por mastectomía parcial, a la resección del tumor con un margen macroscópico de un centímetro y márgenes histológicos idealmente de un centímetro. Es aceptable un margen histológico de hasta $1 \mathrm{ml}$. Para la correcta etapificación de la axila en las pacientes T1N0M0, se recomienda la disección linfática axilar de los niveles I y II (linfonodos ubicados por dorsal y lateral al músculo pectoral menor) o la biopsia del linfonodo centinela axilar, en equipos quirúrgicos entrenados en la técnica. (Paredes H, Baeza R,

\section{Tabla 1. C lasificación T N M cáncer de mama}

Tamaño tumoral (T)

T1: Tumor menor o igual a 2 centímetros

T2: Tumor entre 2-5 centímetros

T3: Tumor mayor de 5 centímetros

T4: Cualquier tamaño, con extensión directa a la piel o pared torácica

Compromiso linfático nodal $(\mathrm{N})$

N0: Sin compromiso metastásico axilar

N1, N2 o N3: Con compromiso metastásico axilar

Según enfermedad a distancia (M)

M0: Sin enfermedad a distancia

M1: Con enfermedad metastásica a distancia 
Horvath E, Torres S. Cáncer de mama en etapas I y II: Cirugía. En: II Jornada de Consenso en Cáncer de mama. Viña de Mar, Chile, septiembre de 2003).

El objetivo del presente trabajo fue revisar nuestra experiencia en una población de pacientes con cáncer de mama precoz, T1N0M0, que recibieron radioterapia adyuvante tras la cirugía, en el Servicio de Radioterapia del Centro de Cáncer Nuestra Señora de la Esperanza, de la Pontificia Universidad Católica de Chile.

Analizamos el motivo de consulta, la toxicidad aguda y tardía de la radioterapia, recurrencias locales (RL), recurrencias a distancia (RD), la sobrevida global (SG) y la sobrevida libre de enfermedad (SLE).

\section{PACIENTES Y MÉTODO}

Es un estudio descriptivo, retrospectivo. Se analizaron las historias clínicas y de radioterapia de pacientes con cáncer de mama infiltrante, de tamaño menor o igual a 2 centímetros, en su diámetro mayor histopatológico; sin compromiso linfático axilar y en ausencia de metástasis (T1N0M0), que fueron sometidas a mastectomía parcial, disección axilar o biopsia del linfonodo centinela axilar y luego radioterapia adyuvante, desde enero de 1997 a mayo de 2004.

La definición del tamaño tumoral, como se expuso anteriormente, y del compromiso linfático axilar fue la determinada por el estudio histopatológico (Clasificación TNM patológica) y no por la etapificación clínica del TNM.

La etapificación sistémica incluyó tomografía axial computada (TAC) de tórax, abdomen y pelvis, además de cintigrama óseo. En algunas pacientes, el estudio se practicó sólo con radiografía de tórax y ecotomografía abdominal.

La radioterapia se administró en el Centro de Cáncer de la Pontificia Universidad Católica de Chile. Se utilizó acelerador lineal de $6 \mathrm{MV}$, recibiendo una dosis de 50 Gy en 25 fracciones. Los pacientes con tumores de grado nuclear alto, 2 y 3, recibieron una sobreimpresión al lecho tumoral de 10 Gy en 5 fracciones.

De las historias clínicas se obtuvieron: edad, motivo de consulta, localización anatómica del tumor e indicación de terapia adyuvante. Se obtuvieron, además, el tamaño tumoral total y del componente infiltrante, tipo histológico, grado nuclear e histológico según la clasificación de Elston y Bloom $^{13,14}$, estudio de receptores de estrógeno (RE) y progesterona (RP), valorado en porcentajes de $0-100 \%$, HER 2 (receptor de factor de crecimiento epidérmico tipo 2) de $+\mathrm{a}+\mathrm{H}$, todos por métodos inmunohistoquímicos. También se consignó el número de linfonodos axilares disecados.

Se definió recurrencia local ipsilateral como la aparición confirmada por biopsia de una recurrencia en el campo de tratamiento de la radioterapia; segundo primario, como la aparición de un tumor en la mama contralateral confirmada por biopsia y recurrencia a distancia como la aparición de metástasis de adenocarcinoma de probable origen mamario fuera del campo de la radioterapia y no en la mama contralateral.

La SLE se definió como el tiempo desde el diagnóstico hasta la confirmación histológica de una recurrencia local, contralateral o a distancia. La SG se calculó desde el momento de diagnóstico hasta la muerte. A las pacientes sin control durante los últimos 6 meses, se les entrevistó telefónicamente para averiguar su estado actual.

La toxicidad aguda de la radioterapia se evaluó semanalmente durante el tratamiento sobre la base de una escala de tres puntos: cambios leves + , cambios moderados + , y cambios severos $H$. Con esta graduación se midió la presencia de eritema, edema, descamación e hiperpigmentación.

La toxicidad tardía se evaluó por cambios en la mama, secuelas pulmonares y cardiacas sintomáticas, observadas en los controles de seguimiento.

El análisis estadístico de sobrevida se hizo utilizando Kaplan Meier. Se utilizó Test de MannWhitney, con intervalos de confianza de 95\%, para comparar el tamaño tumoral de las pacientes que consultaron por síntomas o por hallazgos en la mamografía.

\section{RESULTADOS}

Se analizaron las fichas clínicas de 137 pacientes con cáncer de mama T1N0M0. De 125 fichas, se obtuvo información suficiente para este análisis. Doce pacientes se excluyeron del análisis final: siete por error en la medición del componente infiltrante ( $\mathrm{T}$ mayor de $2 \mathrm{~cm}$ ) y otras 5 por no tener informe histológico disponible. 
El 61\% (76) de las pacientes consultó por alteraciones de la mamografía de control preventivo (screening), 36\% (45) fue evaluada por síntomas o hallazgos al examen físico. En 4 casos no había información.

Las características de las pacientes se resumen en la Tabla 2a. La edad promedio fue de 55 años, con un rango de 35 a 80 años. El 68\% de las pacientes tenía más de 50 años. El $52 \%$ de los tumores se localizaron en la mama izquierda y $48 \%$ en la derecha. A 113/125 (90,4\%) pacientes se les realizó TAC de tórax y a 110/125 (88\%) TAC de abdomen, como parte del estudio inicial, en 98/ $125(78 \%)$ se dispuso de cintigrama óseo inicial.

Las localizaciones más frecuentes eran cuadrante superior externo (CSE) en 54 pacientes (43\%) y cuadrante superior externo más central en 17 pacientes $(13,6 \%)$. Otras localizaciones fueron menos frecuentes. La distribución de los tumores fue similar en la mama derecha e izquierda.

El tamaño tumoral promedio fue de $11,6 \mathrm{~mm}$, 37,6\% (47 tumores) medían 1 centímetro o menos, $38,4 \%$ (48 casos) entre 10,1 y $15 \mathrm{~mm}$ y $24 \%$ (30 casos) más de $15 \mathrm{~mm}$ (Tabla 2b). Si evaluamos el tamaño tumoral del componente infiltrante según el motivo de consulta, vemos que la mediana de los tumores detectados por mamografía fue de 10,9 mm (IC 95\%: 10-12 mm) (Figura 1) y de 14,2 mm (IC 95\%: 12-15 mm) (Figura 2) para aquellos detectados al examen físico ( $\mathrm{P}<0,0157$ ).

Los márgenes quirúrgicos fueron negativos en todos los casos, teniendo un mínimo de $1 \mathrm{~mm}$ en todas las pacientes.

El grado histológico, en su gran mayoría, fue 1 ó 2, y en $14 \%$ grado 3 . El grado nuclear en $55 \%$ de los casos fue informado como grado $1,38 \%$ grado 2 y $7 \%$ grado 3 .

La presencia de RE detectada por métodos inmunohistoquímicos fue positiva en 89/111 (82\%). El 51\% tiene RE superior a $80 \%$ y 5,4\% menor o igual a $10 \%$.

Por otra parte, en 105 pacientes se estudió el estado de los RP, en 76/105 (72,3\%) fue positivo. En $80 \%$ de los casos se realizó estudio de HER2 por método inmunohistoquímico, $68 \%$ del total de biopsias mostró negatividad para HER 2 y sólo 2,4\% se informó como HER 2 +++ (positivo). El estudio de la axila fue negativo en todos los casos, con una mediana de 15 linfonodos disecados.
Tabla 2a. C aracterísticas de las pacientes

$\begin{array}{lr}\text { Número de pacientes } & 125 \\ \text { Edad (años) } & \\ \quad \text { Promedio } & 55 \\ 35-50 & 41 \\ 51-60 & 44 \\ 61-70 & 29 \\ 71-80 & 11 \\ \text { Motivo consulta } & \\ \quad \text { Mamografía } & 76 \\ \text { Examen físico } & 45 \\ \text { Sin información } & 4 \\ \text { Distribución por cuadrantes } & \\ \text { CSE } & 54 \\ \text { CSE+CC } & 17 \\ \text { CSI } & 12 \\ \text { CIE } & 12 \\ \text { CC } & 12 \\ \text { CII } & 9 \\ \text { Otras combinaciones } & 9\end{array}$

CSE: cuadrante supero externo. CSI cuadrante supero interno. CIE: cuadrante ínfero externo. CII: cuadrante ínfero interno. CC: cuadrante central.

Tabla 2b. C aracterísticas de las pacientes

Tamaño tumoral componente infiltrante

0-5 mm

$5,1-10 \mathrm{~mm}$

$10,1-15 \mathrm{~mm}$

$15,1-20 \mathrm{~mm}$

Tipos histológicos

Ductal infiltrante $\quad 91$

Tubular $\quad 12$

Lobulillar $\quad 11$

Mucinoso 3

Medular 2

Combinaciones 6

Estudio de RE

Positivo

89

Negativo

No realizado

14

Mediana de linfonodos disecados 


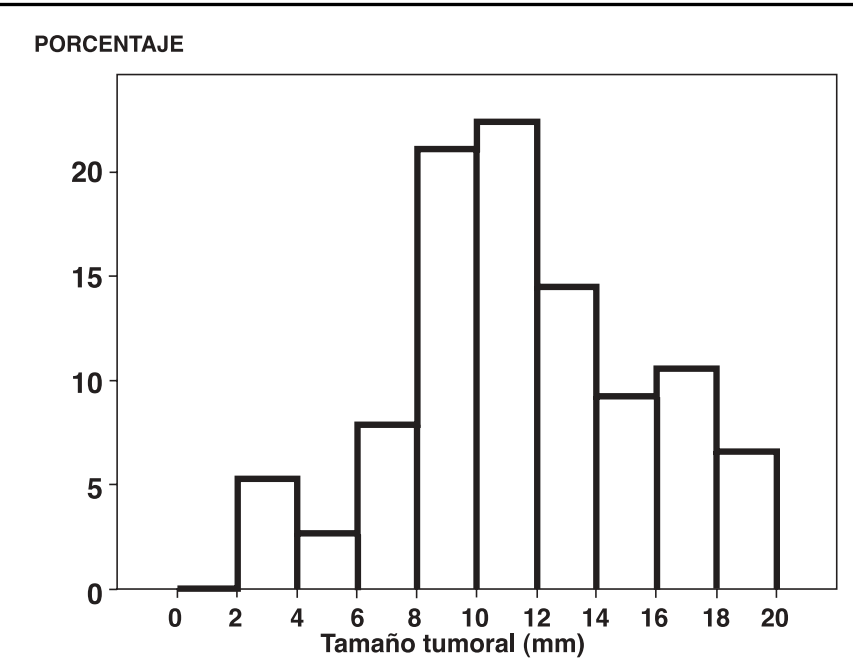

Figura 1. Tamaño tumoral del componente infiltrante en pacientes que consultaron por hallazgos en mamografía de control (screening).

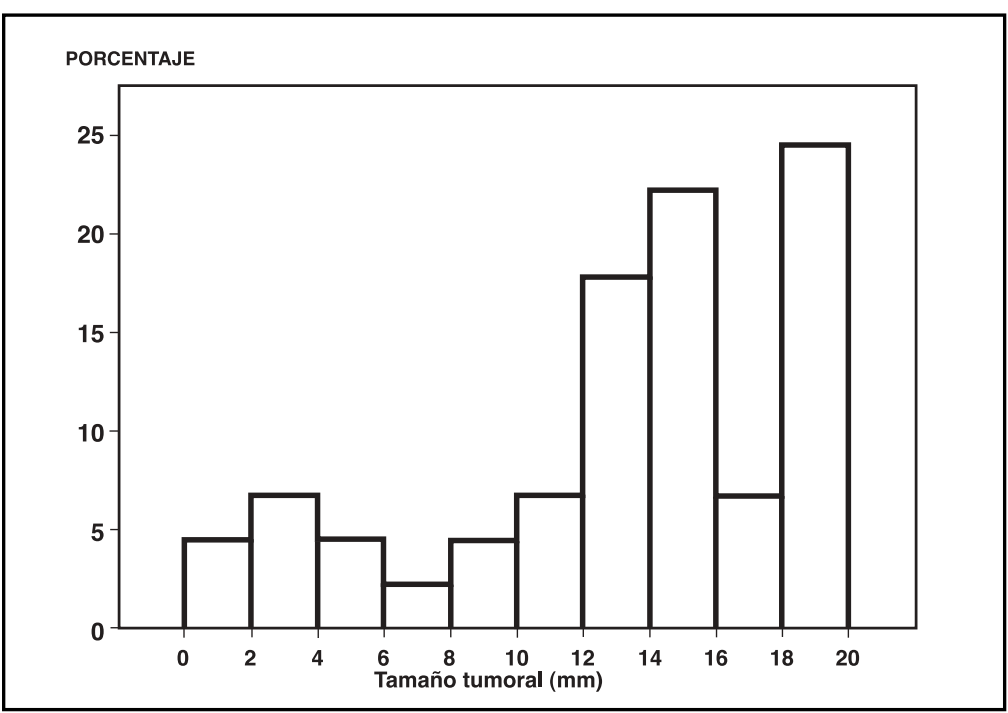

Figura 2. Tamaño tumoral del componente infiltrante en pacientes que consultaron por hallazgos al examen físico.

En 13 casos se realizó sólo estudio de linfonodo centinela.

El intervalo de tiempo promedio entre la cirugía y la radioterapia fue de 36 días. Con respecto a la toxicidad aguda de la radioterapia, encontramos que el hallazgo más frecuente fue el eritema local; leve $(+)$ en $72 \%$ de los casos y severo $(+++)$ en $2,5 \%$ de los casos. La presencia de edema fue el segundo hallazgo más frecuente, severo en $1,5 \%$ de los casos y leve en $46 \%$ de los casos. Ulceración de la piel se presentó en 1,5\% de las pacientes. El tratamiento de radioterapia se completó entre 32 y 46 días. Ninguna paciente suspendió la radioterapia por toxicidad aguda. 
Tabla 3. Características de los 6 pacientes con segundo primario contralateral y recurrencia sistémica

\begin{tabular}{|c|c|c|c|c|c|c|}
\hline \multirow{2}{*}{ Características } & \multicolumn{3}{|c|}{ Segundo primario contralateral } & \multicolumn{3}{|c|}{ Recidiva a distancia } \\
\hline & Paciente 1 & Paciente 2 & Paciente 3 & Paciente 1 & Paciente 2 & Paciente 3 \\
\hline Edad (años) & 54 & 35 & 40 & 37 & 58 & 58 \\
\hline $\begin{array}{l}\text { Tamaño } \\
\text { tumoral (mm) }\end{array}$ & 17,1 & 6,8 & 3,0 & 1,0 & 14,0 & 13,5 \\
\hline $\mathrm{RE}(\%)$ & 90 & 15 & NC & 95 & NC & 95 \\
\hline $\begin{array}{l}\text { Tiempo al evento } \\
\text { (meses) }\end{array}$ & 14,2 & 18,0 & 2,19 & 53,8 & 32,16 & 41,2 \\
\hline $\begin{array}{l}\text { Tratamiento } \\
\text { adyuvante }\end{array}$ & Tamoxifeno & $\begin{array}{l}\text { Sin } \\
\text { tratamiento }\end{array}$ & $\begin{array}{l}\text { Sin } \\
\text { tratamiento }\end{array}$ & Tamoxifeno & $\begin{array}{l}\text { Tamoxifeno y } \\
\text { quimioterapia }\end{array}$ & Tamoxifeno \\
\hline
\end{tabular}

RE: estudio receptores estrógenos por inmunohistoquímica. NC: desconocido.

La toxicidad tardía fue principalmente hiperpigmentación mamaria, que desapareció gradualmente. No se ha producido ningún caso de toxicidad pulmonar o cardiaca sintomática hasta la fecha.

$\mathrm{Al}$ evaluar el uso de terapia adyuvante, observamos que a $80 \%$ de las pacientes se les indicó tratamiento con tamoxifeno por 5 años y en $14 \%$ de los casos se utilizó quimioterapia adyuvante.

A un seguimiento promedio de 40,42 meses no se presentaron recurrencias ipsilaterales, 3 pacientes $(2,4 \%)$ presentaron segundos primarios contralaterales y 3 (2,4\%) desarrollaron enfermedad sistémica. Dos de estas últimas pacientes $(1,26 \%)$ han fallecido a consecuencia de su cáncer. En la Tabla 3 se presentan las características y tiempo a la recurrencia de estas 6 pacientes. La SLE a 3 años fue de 95\% (Figura 3), con una SG de 96,5\% (Figura 4).

\section{DisCUSIÓN}

Nuestra serie presenta los resultados del tratamiento del cáncer de mama diagnosticado en su etapa inicial. El diagnóstico precoz se logró

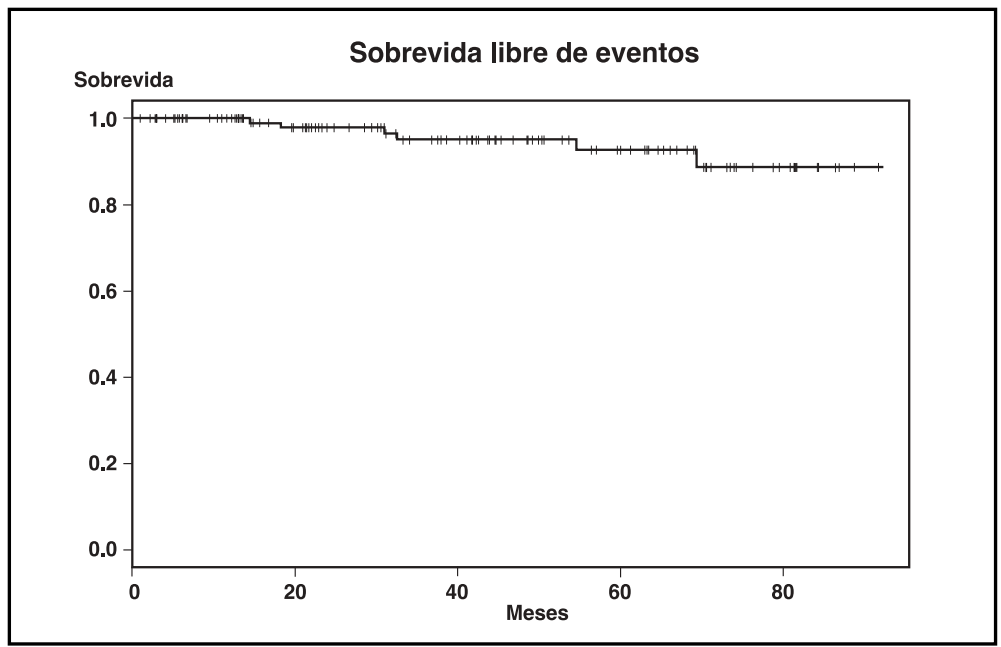

Figura 3. Sobrevida libre de enfermedad de 125 pacientes con cáncer de mama T1N0M0, tratados con mastectomía parcial y radioterapia adyuvante. 


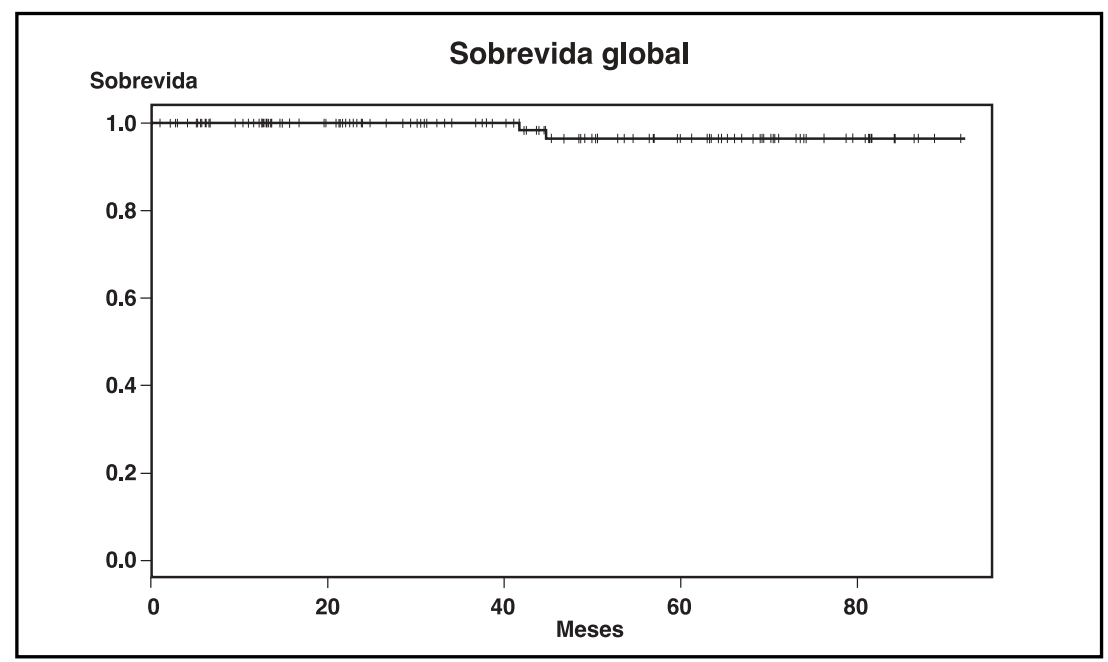

Figura 4. Sobrevida global de 125 pacientes con cáncer de mama T1N0M0, tratados con mastectomía parcial y radioterapia adyuvante.

mediante el estudio con mamografía de control preventivo en $61 \%$ de los casos, siendo estos tumores de menor tamaño que los detectados clínicamente. Nuestros resultados apoyan la necesidad del control periódico con mamografía, para obtener un diagnóstico precoz del cáncer de mama y lograr su curación. Al igual que en series anteriores, la localización más frecuente del cáncer de mama fue en el cuadrante superior externo, en $56 \%$ y se distribuyeron casi por igual en ambas mamas derecha e izquierda. La histología más frecuente fue también el carcinoma ductal infiltrante, con $73 \% 15,16$. Alrededor de $90 \%$ de las pacientes se estudiaron con TAC de tórax, abdomen y pelvis, asegurando a lo menos radiológicamente, la ausencia de enfermedad a distancia (M0); sin embargo, en pacientes con CM en etapas precoces, las recomendaciones de etapificación de la enfermedad no incluyen la evaluación con TAC, dada la baja probabilidad de encontrar enfermedad sistémica ${ }^{17}$.

El tratamiento con radioterapia se efectuó en forma completa y sin retrasos con toxicidad aguda menor. No hemos encontrado hasta el momento complicaciones respiratorias y cardíacas sintomáticas. Una paciente desarrolló linfedema del brazo que fue atribuible a la cirugía axilar. La radioterapia es, en general, un tratamiento bien tolerado ${ }^{18}$; sin embargo, en trabajos clásicos, el grupo sometido a radioterapia ha mostrado una mayor mortalidad a largo plazo por enfermedad cardiovascular ${ }^{19,20}$. Estas series utilizaron técnicas antiguas de radioterapia. La radioterapia moderna con acelerador lineal, inmovilización, planificación y dosimetría adecuados, debe evitar estas complicaciones. Con un mayor seguimiento podremos definir en nuestra serie la toxicidad a largo plazo.

Estudios recientes plantean el uso de irradiación parcial en el cáncer de mama, pero con una mayor incidencia de complicaciones y recidiva local ${ }^{21,22}$.

El control de la enfermedad en la mama irradiada ha sido óptimo, no demostrándose ninguna recidiva local con más de 3 años de seguimiento. De acuerdo con la literatura, la recidiva local ipsilateral esperada es de $3 \%$ a $5 \%$ para este periodo ${ }^{23}$. Múltiples factores de nuestra serie influyen en los resultados: márgenes quirúrgicos negativos, grado histológico, estado de receptores hormonales, tratamiento adyuvante; pero también es de gran importancia el uso de radioterapia moderna, con distribución de dosis adecuadas y tratamientos sin suspensiones.

A pesar de que $89 \%$ de las pacientes tenían presencia de RE por inmunohistoquímica en el tejido tumoral, sólo $80 \%$ recibió la indicación de 
tamoxifeno. La naturaleza retrospectiva de este trabajo impide evaluar con certeza los motivos para esta diferencia. Una razón puede ser la evaluación individual de la relación riesgo/beneficio que no favorecía la indicación de tamoxifeno (pacientes con mayor riesgo de cáncer de endometrio, eventos embólicos, cataratas, daño hepático, etc.) en este grupo de pacientes.

Dado el pequeño número de recurrencias, no es posible analizar en esta serie los factores asociados-predictivos a una recaída de la enfermedad. Debido a la dificultad de establecer el pronóstico individual de una paciente con cáncer de mama precoz, y como forma de ayudar en la toma de decisiones, Ravdin et al proponen un sistema computacional para definir el riesgo de un paciente individual ${ }^{24}$. Muchos estudios han examinado potenciales factores pronósticos en cáncer de mama invasivo, pero pocos estudios incluyen a

\section{REFERENCIAS}

1. Aapro M. Adyuvant therapy of primary breast cancer: a review of key findings from the $7^{\text {th }}$ International Conference, St. Gallen, february 2001. The Oncologist 2001; 6: 376-85.

2. Jemal A, Siegel R, Ward E, Murray $T, X_{u}$ J, Smigal $C$ ET AL. Cancer Statistics. CA Cancer J Clin 2006; 56: 106-30.

3. MINSAL, Ministerio de Salud de Chile (www.minsal.cl)

4. Orellana C, Torres S, Derio L, Prieto M. Cancer care in Chile. Lancet Oncol 2003; 4: 653-6.

5. Cady B, Stone M JG, Thakur R, Wanner MA, Lavin PT. The new era in breast cancer: Invasion, size, and nodal involvement dramatically decreasing as a result of mammographic screening. Arch Surg 1996; 131: 301-8.

6. FisHER B. Cancer of the breast: size of neoplasm and prognosis. Cancer 1969; 24: 1071-80.

7. Joensuu $\mathrm{H}$, ToikKanen S. Comparison of breast carcinomas diagnosed in the 1980's with diagnosed in the 1940 to 1960. Br Med J 1991; 303: 155-8.

8. Yarbro J, Page D, Fielding L American Joint Committee on Cancer Prognostic Factors Consensus Conference. Cancer 1999; 86: 2436-46. pacientes sin compromiso linfático y menos aún de bajo riesgo ${ }^{25}$. La evaluación de marcadores biológicos pronósticos ha permitido establecer nuevos subgrupos pronósticos, que necesitan ser validados en estudios prospectivos ${ }^{1,26-30}$.

En conclusión, esta serie muestra el buen control local del cáncer de mama en etapas precoces con mínima toxicidad utilizando radioterapia moderna. Sin duda es necesario un tiempo más largo de evaluación para detectar recurrencias tardías y complicaciones terapéuticas.

El cáncer de mama tiene una baja mortalidad y es curable en etapas precoces, lo que refuerza la necesidad del diagnóstico precoz y el uso del control preventivo con mamografía.

Debemos buscar mejores alternativas de tratamientos y algoritmos para usar terapias individualizadas y así evitar recurrencias de la enfermedad, aun en pacientes de bajo riesgo.

9. Singletary E, Aurred C, Ashley P, Bassett L, Berry D, KIRBY I ET AL. Revision of the American Joint Committee on Cancer Staging System for Breast Cancer. J Clin Oncol 2002; 20: 3628-36.

10. Valagussa P, Bonadonna G, Veronesi U. Patterns of relapse and survival following radical mastectomy. Analysis of 716 consecutive patients. Cancer 1978; 41: 1170-8.

11. Vinh-Hung V, Verschraegen C. Breast-Conserving Surgery With or Without Radiotherapy: PooledAnalysis for Risks of Ipsilateral Breast Tumor Recurrence and Mortality. J Natl Cancer Inst 2004; 96: 115-21.

12. Fisher B, Anderson S, Bryant J, Margolese RG, Deutsch M, Fisher ER ET AL. Twenty-year follow-up of a randomized trial comparing total mastectomy, lumpectomy, and lumpectomy plus irradiation for the treatment of invasive breast cancer. $\mathrm{N}$ Engl J Med 2002; 347: 1233-41.

13. ELSTON CW, EUS IO. Pathological prognostic factors in breast cancer. I. The value of histological grade in breast cancer: experience from a large study with long-term follow-up. Histopathology 1991; 19: 403-10.

14. BLоom HJG, RichaRdson WW. Histological grading and prognosis in breast cancer. A study of 1409 
cases of wich 359 have been followed for 15 years. Br J Cancer 1957; II: 359-77.

15. Rosen PP, Groshen S, KinNe D, Norton L. Factors influencing prognosis in node - negative breast carcinoma: analysis of 767 T1N0M0/T1N0M0 patients with a long follow-up. J Clin Oncol 1993; 11: 2090-100.

16. SchnitT SJ, Guidi AJ. Pathology and biological markers of invasive breast cancer. Pathology of invasive breast cancer. En: Harris JR, Lippman ME, Morrow M, Osborne CK, eds. Diseases of the Breast, $2^{\text {nd }}$ edition. Philadelphia: Lippincott, Williams and Wilkins, 2000; 425-70.

17. National Comprehensive Cancer Network. Clinical Practice Guidelines in Oncology. http:// www.nccn.org/professionals/physician_gls/PDF/ breast.pdf. Visitado el 9-06-06.

18. Whelan TJ, Levine M, Julian J, Kirkbride P, Skingley $P$. The effects of radiation therapy on quality of life of women with breast carcinoma: results of a randomized trial. Ontario Clinical Oncology Group. Cancer 2000; 88: 2260-6.

19. Paszat LF, Mackiшop WJ, Groome PA, Schuize K, HoLowaTY E. Mortality from myocardial infarction following postlumpectomy radiotherapy for breast cancer: a population-based study in Ontario, Canada. IntJ Radiat Oncol Biol Phys 1999; 43: 755-62.

20. Vauis KA, PintiLe M, Chong N, Holowaty E, Douglas PS, KirKBRide P ET AL. Assessment of coronary heart disease morbidity and mortality after radiation therapy for early breast cancer. J Clin Oncol 2002; 20: 1036-42.

21. Coles C, Moody A, Wilson C, Burnet N. Reduction of radiotherapy-induced late complications in early breast cancer: the role of intensity-modulated radiation therapy and partial breast irradiation. Part II-Radiotherapy strategies to reduce radiation-induced late effects. Clin Oncol (R Coll Radiol) 2005; 17: 98-110.
22. Coles C, Moody A, Wilson C, Burnet N. Reduction of radiotherapy-induced late complications in early breast cancer: the role of intensity-modulated radiation therapy and partial breast irradiation. Part I-normal tissue complications. Clin Oncol (R Coll Radiol) 2005; 17: 16-24.

23. Krauss D, Kestin L, Mitchell C, Martínez A, Vicin F. Changes in temporal patterns of local failure after breast-conserving therapy and their prognostic implications. Int J Radiation Oncology Biol Phys 2004; 60: 731-40.

24. Ravdin P, Siminoff L, Davis G, Mercer M, Hewlett J, Gerson N et aL. Computer program to assist in Making Decisions About Adjuvant Therapy for Women With Early Breast Cancer. J Clin Oncol 2001; 19: 980-91.

25. Sánchez C, Galndo H, León A, Alvarez M. Rol de la quimioterapia en cáncer de mama T1N0M0. Rev Chilena de Cirugía 2004; 56: 495-502.

26. Mirza A, Mirza N, Vlastos G, Singletary S. Prognostic factors in node-negative breast cancer: a review of studies with sample size more than 200 and follow-up more than 5 years. Annals of Surgery 2002; 235: 10-26.

27. Goldhirsh A, Glick J, Gelber R, Coates A, Senn H. Meeting Highlights: International consensus panel on the treatment of primary breast cancer. J Clin Oncol 2001; 19: 3817-27.

28. Van De Vijver MJ, He YD, Van't Veer LJ, Dai H, HaRt AA, Voskull DW et al. A gene-expression signature as a predictor of survival in breast cancer. N Engl J Med 2002; 347: 1999-2009.

29. Perou C, Sorle T, Eisen B, Van De Rujn M, Jefrerey, REES CA ET AL. Molecular portraits of human breast tumours. Nature 2000; 406: 747-52.

30. Pusztai L, Ayers M, Stec J, Hortobagyi G. Clinical application of cDNA microarrays in Oncology. The Oncologist 2003; 8: 252-8. 